\title{
OBTENÇÃO E CARACTERIZAÇÃO DE NANOFIBRAS DE POLI(ACRILONITRILA-CO-ACETATO DE VINILA) OBTIDAS POR ELETROFIAÇÃO*
}

\author{
Jorge Costa Silva Fllho ${ }^{1}$ \\ Giulia Maria Rodrigues Alvares ${ }^{2}$ \\ Daniel Felipe Simão ${ }^{3}$ \\ Marcia Tsuyama Escote 4 \\ Everaldo Carlos Venancio ${ }^{5}$
}

\section{Resumo}

Neste trabalho é apresentado o estudo sobre a obtenção de nanofibras de poli(acrilonitrila-co-acetato de vinila) (PANVA). Com o desenvolvimento da nanociência e da nanotecnologia; novos métodos de processamento de PANVA podem ser utilizados para a obtenção de nanofibras e microfibras de PANVA. Neste trabalho, as nanofibras de PANVA foram obtidas por meio do uso da técnica de eletrofiação. Os resultados de microscopia eletrônica de varredura mostraram a obtenção de nanofibras de PANVA com diâmetro médio de $100 \mathrm{~nm}$. Estas amostras foram submetidas a um tratamento termooxidativo a diferentes temperaturas, 220,240 e $260^{\circ} \mathrm{C}$, sob atmosfera de oxigênio. Os resultados mostraram alterações nas características da manta obtida, indicando a possível ocorrência do processo de formação de estruturas cíclicas a partir da unidade de acrilonitrila presente na estrutura da PANVA.

Palavras-chave: Nanofibras; Eletrofiação; Poliacrilonitrila; Poli(acrilonitrila-coacetato de vinila.

\section{PREPARATION AND CHARACTERIZATION OF POLYACRYLONITRILE FIBERS OBTAINED BY ELECTROSPINNING}

\section{Abstract}

In this work is presented the results related to the obtention of poly(acrylonitrile-covinyl acetate) (PANVA). The development of the nanoscience and nanotechnology, new PANVA processing methods can be used to obtain PANVA nanofibers and microfibes. In this work, the PANVA nanofibers were obtained by using the electrospinning technique. The SEM results showed PANVA nanofibers with mean diameter of $\sim 100 \mathrm{~nm}$. These samples were submitted to a thermoxidative treatment using differente tempratures, i.e. 220,240 and $260^{\circ} \mathrm{C}$, under oxygen atmosphere. The PANVA nanofiber samples showed changes in their physical charcteristics, which might indicate the cyclization process occurred from the acrylonitrile units present in the PANVA structure.

Keywords: Nanofibers; Electrospinning; Polyacrylonitrile; Poly(acrylonitrile-co-vinyl acetate).

1 Engenharia de Materiais, Bacharel, estudante, Universidade Federal do ABC, Santo André, Sâo Paulo, Brasil.

2 Tecnóloga em Polímeros, Mestre, aluna de pós-graduação, Universidade Federal do ABC,

3 Físico, licenciatura, Mestre, aluno de pós-graduação, Universidade Federal do ABC, Santo André, São Paulo, Brasil

4 Física, Pós Doutora, professora, Engenharia de Materiais, Centro de Engenharia Modelagem e Simulação, Universidade Federal do ABC, Santo André, São Paulo, Brasil

5 Químico, Pós Doutor, professor, Engenharia de Materiais, Centro de Engenharia Modelagem e Simulação, Universidade Federal do ABC, Santo André, São Paulo, Brasil. 


\section{INTRODUÇÃO}

As fibras de carbono (CFs) são, talvez, o material a base de carbono de maior sucesso a ser comercializado nos últimos anos. A sua elevada resistência e rigidez, combinada com o fato de ser um material leve, fazem essas fibras atraentes para aplicações que vão desde artigos esportivos até estruturas de aeronaves. Hoje, fibras de carbono também estão sendo desenvolvidas para outras formas de aplicação, como por exemplo, gerenciamento térmico. Como outros produtos, fibras de carbono comerciais devem apresentar propriedades mecânicas e de transporte consistentes, e as propriedades podem ser diferentes para cada aplicação. Devido a isso, o controle da estrutura e da interação entre a estrutura e propriedades tem sido extensivamente estudado nas fibras de alto desempenho [1].

Embora o progresso considerável tenha ocorrido ao longo dos últimos 35 anos, a origem e o desenvolvimento da estrutura, bem como a relação entre a estrutura e as propriedades ainda não estão completamente esclarecidos para fibras de carbono. Portanto, para compreender a estrutura e as propriedades destas CFs, é necessário um detalhamento dos materiais e processos utilizados para formar as fibras [2]. A produção de nanofibras de carbono (CNFs) a partir da poliacrilonitrila (PAN) por meio do uso da técnica de eletrofiação vem despertando interesse [3,4]. As técnicas tradicionais utilizadas para a produção de diferentes formas fibras de carbono permitem obter fibras com diâmetros médios da ordem de $1,5 \mathrm{~nm}[5,6]$ a até $500 \mu \mathrm{m}$ [7]. Por outro lado, a eletrofiação é uma técnica para a fabricação de fibras com diâmetros que variam da escala manométrica a até a escala micrométrica $[8,9]$.

De 1934 a 1944, Formhals depositou uma série de patentes descrevendo o aparato experimental para a produção de filamentos poliméricos usando força eletrostática $[10,11]$. O processo de eletrofiação via solução polimérica consiste na aplicação de forças eletrostáticas e de arraste na solução resultando na formação de uma gota contendo a solução polimérica, e a partir desta gota ocorre a formação de nanofibras. Estudos sobre eletrofiação envolvem tipicamente polímeros de alta massa molar média devido às cadeias poliméricas sofrerem emaranhamentos e este ser um fator importante para que ocorra a formação de fibras eletrofiadas. Entretanto, trabalhos recentes mostraram que a alta massa molar média do polímero não é um fator determinante para a obtenção de fibras eletrofiadas, e sim a presença de suficientes interações intermoleculares, que proporcionam um substituto para a conectividade intercadeias por meio do emaranhamento das fibras [12].

Neste trabalho foram produzidas nanofibras de poli(acrilonitrila-co-acetato de vinila) (PANVA) por meio do uso da técnica de eletrofiação. As nanofibras obtidas foram submetidas a um processo de estabilização termooxidativo em atmosfera oxidante na presença de oxigênio do ar.

\section{MATERIAIS E MÉTODOS}

As nanofibras de PANVA forma obtidas por meio do uso da técnica de eletrofiação. A concentração de PANVA utilizada foi de $6 \%$ (massa/volume, $\mathrm{m} / \mathrm{v}$ ) utilizando-se a N,N-dimetilformamida (DMF, Sigma Aldrich) como solvente. A amostra de PANVA, contendo $94 \%$ de acrilonitrila e $6 \%$ de acetato de vinila, foi gentilmente cedida pela QuimLab Produtos de Química Fina Ltda. As condições experimentais de eletrofiação utilizadas foram: vazão utilizada, $1 \mathrm{~mL} / \mathrm{h}$; distância de trabalho, $10 \mathrm{~cm}$; tensão DC aplicada, $20 \mathrm{kV}$. A temperatura e a umidade relativa no interior do 
sistema de eletrofiação foram $28 \pm 2{ }^{\circ} \mathrm{C}$ e $56 \pm 2 \%$, respectivamente. O coletor utilizado foi uma folha de alumínio. O diâmetro da agulha de aço utilizada foi de 0,70 mm (BD do Brasil). O sistema de eletrofiação utilizado está apresentado na Figura 1 [13].

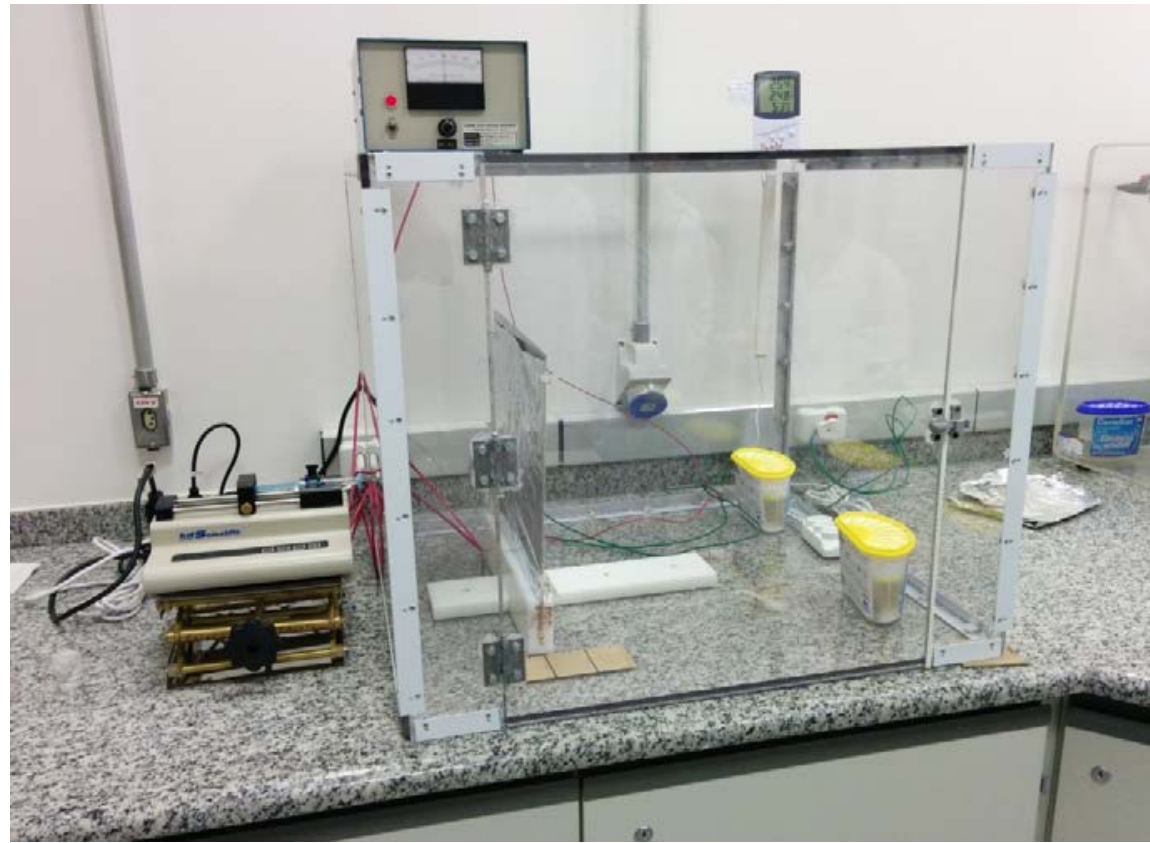

Figura1: Sistema de eletrofiação desenvolvido pelo grupo de pesquisa e utilizado neste trabalho [13].

Os experimentos de microscopia eletrônica de varredura (MEV) foram realizados sem a utilização de recobrimento metálico das amostras. O equipamento utilizado foi um microscópio eletrônico JMS-6701F da JEOL. A tensão aplicada para a extração de elétrons utilizada foi de $5,0 \mathrm{kV}$. A tensão de aceleração de elétrons utilizada foi de $2,0 \mathrm{kV}$.

O tratamento termooxidativo foi realizado utilizando-se um forno tubular LINDBERG/Blue $\mathrm{M}$ da Thermo Electron Corp. com uma rampa de temperatura de $10^{\circ} \mathrm{C} /$ minuto, ao ar em um tubo de alumina. As temperaturas utilizadas foram 220 , 240 e $260^{\circ} \mathrm{C}$, com tempos de 30 e 90 minutos.

\section{RESULTADOS E DISCUSSÃO}

Na Figura 2 são apresentadas as imagens de MEV das amostras de PANVA eletrofiadas com um tempo de eletrofiação de 1 hora (Fig. 2a-b) e 2 horas (Fig. 2c). As imagens de MEV apresentadas na Fig. 2a-b apresentam morfologia fibrilar, com diâmetro médio da ordem de $100 \mathrm{~nm}$. Na Fig. 2c é apresentada a imagem de MEV da amostra de PANVA obtida com tempo de eletrofiação de 2 horas, onde pode ser observada uma morfologia fibrilar, com diâmetro médio da ordem de $100 \mathrm{~nm}$. Estes resultados mostraram que o aumento do tempo de eletrofiação apenas aumentou a quantidade de nanofibras depositadas no coletor.

A amostra 3 (figuras 5) apresenta imagem de MEV para nanofibras de poliacrilonitrila preparadas por meio de aplicação de potencial de tensão de $20 \mathrm{kv}$ $(1.0 \mathrm{kV} / \mathrm{cm})$, esta amostra foi eletrofiada com uma distância de trabalho de $10 \mathrm{~cm}$, e uma vazão de $1 \mathrm{ml} / \mathrm{h}$, por um período de duas horas. Á temperatura externa ao equipamento de $31^{\circ} \mathrm{C}$, temperatura interna do equipamento de $29,9^{\circ} \mathrm{C}$ e com uma umidade relativa do ar de $60 \%$. 


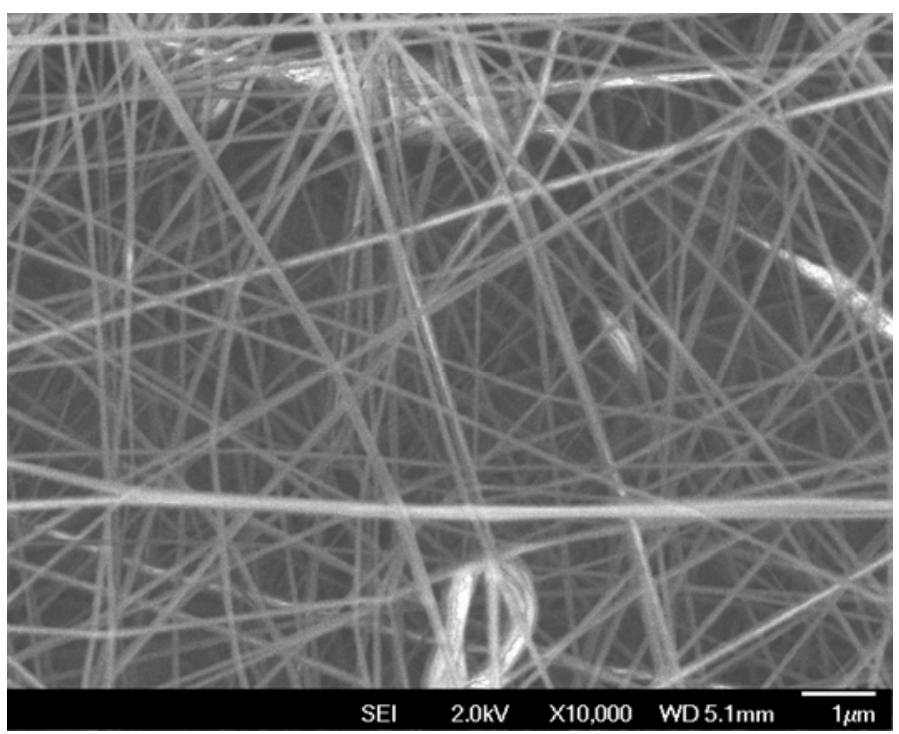

(a)

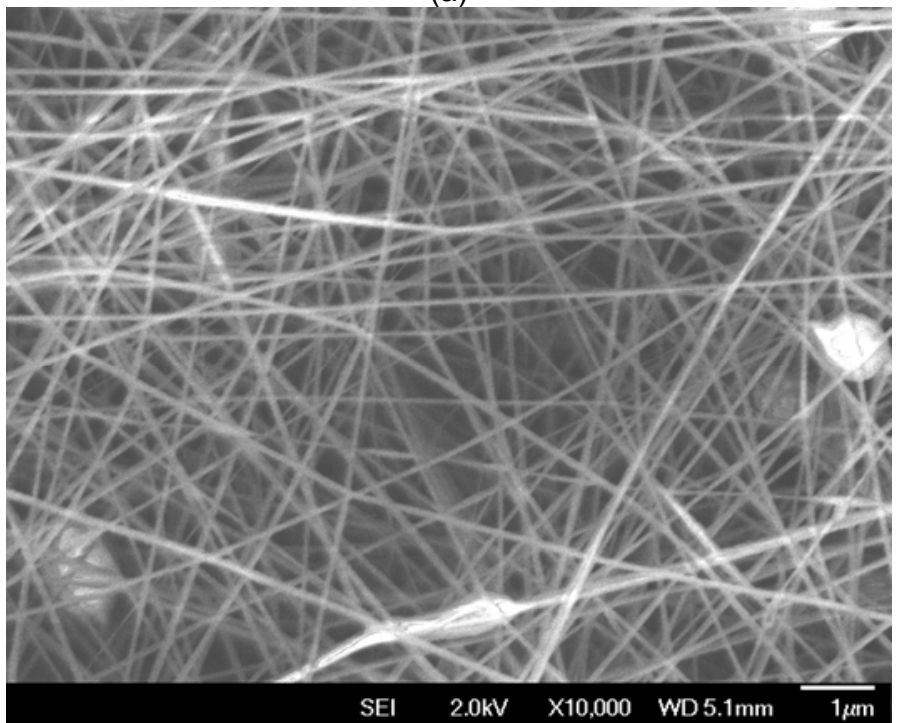

(b)

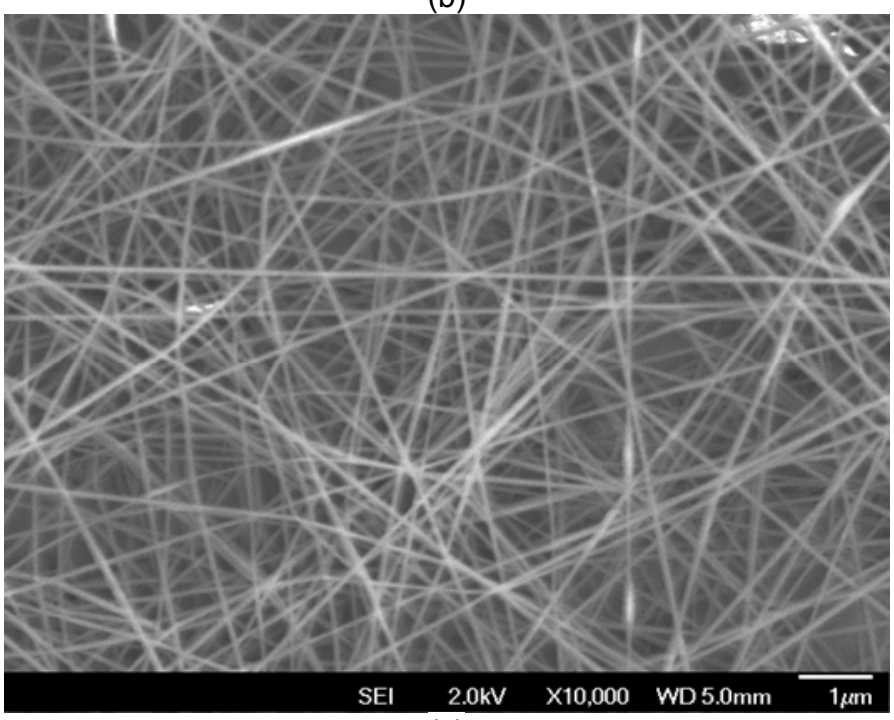

(c)

Figura 2: Imagens de MEV da amostras de nanofibras de PANVA eletrofiadas com diferentes tempos de eletrofiação: (a) amostra 1, 1 hora; (b) amostra 2, 1 hora; (c) amostra 3, 2 horas. 
Na Figura 3 é apresentada uma imagem da amostra de PANVA depositada no substrato de alumínio (Fig. 3a), amostra autossustentável (Fig. 3b), que foi removida do substrato de alumínio, e as amostras submetidas a diferentes tratamentos térmicos termooxidativos, $220^{\circ} \mathrm{C}$ (Fig. 3c) e $260^{\circ} \mathrm{C}$ (Fig. 3d), ambas tratadas durante 90 minutos (Fig. 3c-d), respectivamente. Estas amostras referem-se a amostra de nanofibras de PANVA obtidas de acordo com o descrito para a Figura 2c. Os resultados mostram que após o tratamento térmico é visível a transição da cor branca inicial para uma cor escura, acentuando-se para a amostra tratada durante 90 minutos a $260^{\circ} \mathrm{C}$. Esta transição de coloração da amostra é um indicativo da ocorrência de um processo de formação de estruturas cíclicas a partir da base de acrilonitrila presente na amostra de PANVA.
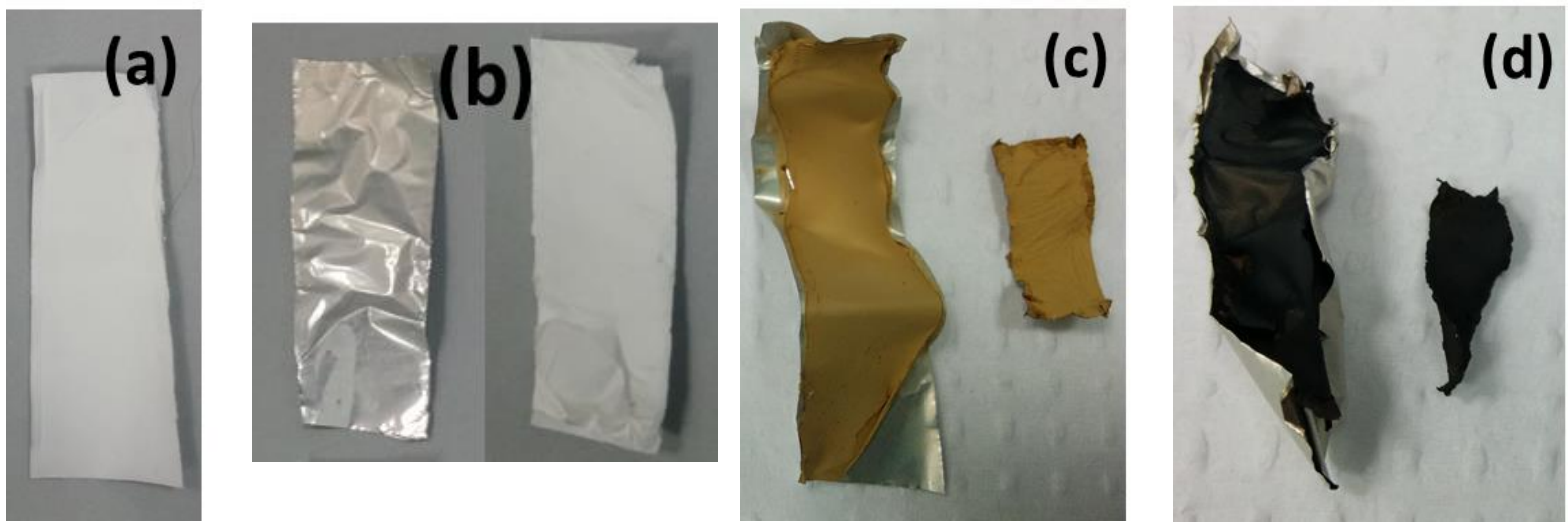

Figura 3: Imagens das amostras de nanofibras de PANVA eletrofiadas durante 2 horas. Amostras sem tratamento termooxidativo, (a) amostra de PANVA depositada sobre o substrato de alumínio e (b) amostra removida do substrato de alumínio. Amostras submetidas ao tratamento termooxidativo durante 90 minutos na presença de oxigênio do ar, (c) $220^{\circ} \mathrm{C}$ e (d) $260^{\circ} \mathrm{C}$, respectivamente.

\section{CONCLUSÃO}

Os resultados apresentados mostraram a obtenção de nanofibras de PANVA com diâmetro médio $\sim 100 \mathrm{~nm}$. As nanofibras obtidas são uniformes, resultando na obtenção de mantas autossustentáveis. O tratamento termooxidativo resultou em alteração da amostra, passando de uma cor branca para uma cor escura, sendo um indicativo de ocorrência do processo de formação de estruturas cíclicas a partir das unidades de acrilonitrila presentes na PANVA. A caracterização da morfologia da amostra tratada por meio de MEV e estrutural por meio de espectroscopia de absorção na região do infravermelho está sendo realizada e será utilizada para confirmar o processo de formação de estruturas cíclicas. Estes materiais serão utilizados como percursores para a produção de fibras de carbono, as quais tem potencial para aplicação em materiais compósitos estruturais e funcionais.

\section{Agradecimentos}

Ao CNPq (Processo Universal número 481647/2011-2), à Central Experimental Multiusuário (CEM-UFABC) e à Universidade Federal do $A B C$ (UFABC).

\section{REFERÊNCIAS}

1 Pamula E, Rouxhet PG. Bullk and surface chemical functionalities of type III PAN-based carbon fibres, Carbon. 2003;(41):1905-1915. 
2 Edie DD. The effect of processing on the structure and properties of carbon fibers, Carbon. 2012;36(4):345-362.

3 Nataraj SK, Yang KS, Aminabhavi TM. Polyacrylonitrile-based nanofibers- A state-ofthe-art review, Progress in Polymer Science. 2012;(37):487-513.

4 Arshad SN, Naraghi M, Chasiotis I. Strong carbon nanofiber from electrospun polyacrylonitrile, Carbon. 2011;(49):1710-1719.

5 Nadarajah A, Lawrence JG. Development and commercialization of vapor grown carbon nanofibers: a review, Key Eng. Materials. 2008;(380):193-206.

6 Ren ZF, Huang ZP, Xu JW, Wang JH, Bush P, Siegal MP, Provencio PN. Synthesis of large arrays of well-aligned carbon nanotubes on glass, Science. 1998;(282):282:105.

7 Park JS, Park JW, Ruckenstein E. Thermal and dynamic mechanical analysis of PVA/MC blend hydrogels, Polymer. 2001;(42):4271-4280.

8 Boland ED, Wnek GE, Simpson DG, Pawloski KJ, Bowlin GL. Tailoring Tissue Engineering Scaffolds Using Electrostatic Processing Techiques: a study of poly(glycolic acid) electrospinning. J. Macromol. Sci, Part A: Pure Appl. Chem. 2001;A38(12):1231-1243.

9 Eichhorn SJ, Sampson WW. Statistical Geometry of Pores and Statistics of Porous Nanofibrous Assemblies, J. R. Soc. Interface. 2005;2(4):309-318

10 Formhals A. United States Patent 1975504, Process and apparatus for preparing artificial threads, 1934.

11 Formhals A. United States Patent 2349950, Method and apparatus for spinning, 1944.

12 Mckee MG, Layman JM, Cashion MP, Long TE. Phospholipid Nonwoven Electrospun Membranes, Science. 2006;31(5759):311-353.

13 Alavares GMR. Desenvolvimento e Caracterização de Fibras de Poli(acrilonitrila-coacetato de vinila) Obtidas Por Meio de Eletrofiação [dissertação de mestrado]. Santo André: Universidade Federal do ABC (UFABC); 2015. 